\title{
THE POISSON KERNELS AND TंHE CAUCHY PROBLEM FOR ELLIPTIC EQUATIONS WITH ANALYTIC COEFFICIENTS
}

\author{
BY \\ YOSHIO KATO
}

1. Introduction. It is well known that the Cauchy problem for elliptic equations with $C^{\infty}$-data cannot always be solved, even if their coefficients are analytic and the problem is local. In this paper we shall discuss relations between Cauchy data with which the Cauchy problem has at least a local solution. Obviously one of the sufficient conditions is that Cauchy data are all analytic (the Cauchy-Kowalewski theorem). In [2] J. Hadamard emphasizes that in order that the Cauchy problem for the laplacian $\Delta$ have a solution, it is necessary for the Cauchy data to satisfy some relation, and, in fact, states such a relation for $\Delta$. Our result is an extension of that for $\Delta$ to general linear elliptic operators.

Let $\Omega$ be a bounded domain containing in the half space $R_{+}^{n+1}$ of the $(n+1)$ dimensional euclidian space $R^{n+1}(n \geqq 1)$ with coordinates $x_{1}, \ldots, x_{n}, t$ defined by the relation $t>0$. We assume that the boundary of $\Omega, \partial \Omega$, is of class $C^{\infty}$ (for the definition see $\$ 2$ ) and contains a domain $\omega$ of the hyperplane $t=0$. By $\alpha$ we denote multi-indices $\left(\alpha_{1}, \ldots, \alpha_{n}\right)$ of nonnegative integers. Their sum is denoted by $|\alpha|$. With $D_{j}=\partial / \partial x_{j}$ and real numbers $\xi_{j}$, we set $D_{x}=\left(D_{1}, \ldots, D_{n}\right), D_{x}^{\alpha}=D_{1}^{\alpha}{ }_{1} \ldots D_{n}^{\alpha}$ and $\xi^{\alpha}=\xi_{1}^{\alpha} \cdots \xi_{n^{n}}^{\alpha}$

Consider a linear partial differential operator of even order

$$
L\left(x, t ; D_{x}, D_{t}\right)=\sum_{|\alpha|+k \leqq 2 m} a_{\alpha k}(x, t) D_{x}^{\alpha} D_{t}^{k},
$$

where $D_{t}=\partial / \partial t$ and $a_{\alpha k}(x, t)$ are complex-valued analytic functions in a domain containing the closure of $\Omega, \bar{\Omega}$. We suppose further that $L$ is properly elliptic in $\bar{\Omega}$, i.e., if $l\left(x, t ; D_{x}, D_{t}\right)$ is the part of order $2 m$ of $L\left(x, t ; D_{x}, D_{t}\right)$, then for every real $(n+1)$-vector $\left(\xi_{1}, \ldots, \xi_{n}, \tau\right) \neq 0$ and for every point $(x, t)$ in $\bar{\Omega}$, we have $l(x, t ; \xi, \tau) \neq 0$, and for every $\xi \neq 0$ and for every $(x, t) \in \bar{\Omega}$ the polynomial of $\tau, l(x, t ; \xi, \tau)$, has exactly $m$ roots $\tau_{1}(x, t ; \xi), \ldots, \tau_{m}(x, t ; \xi)$ with positive imaginary parts. It is clear that every elliptic operator is properly elliptic if $n \geqq 2$.

Consider next the set of linear partial differential operators with coefficients in $C^{\infty}(\partial \Omega)(\partial \Omega=\bar{\Omega}-\Omega)$

$$
B_{j}\left(x, t ; D_{x}, D_{t}\right)=\sum_{|\alpha|+k \leqq \mu_{j}} b_{j, \alpha k}(x, t) D_{x}^{\alpha} D_{t}^{k}, \quad j=1, \ldots, 2 m,
$$

Received by the editors December 26, 1968. 
where $\mu_{j}$ are exact order of $B_{j}$ and $\mu_{j}<2 m$. We assume that $\left\{B_{j}\right\}_{j=1}^{2 m}$ is a Dirichlet set, i.e., (i) $\mu_{j} \neq \mu_{k}$ for $j \neq k$ and (ii) if $b_{j}(x, t ; \xi, \tau)$ is the part of order $\mu_{j}$ of $B_{j}$, then we have $b_{j}(x, t ; \boldsymbol{n}) \neq 0$ when $\boldsymbol{n} \neq 0$ is normal to $\partial \Omega$ at $(x, t)$, and that the set $B_{1}, \ldots, B_{m}$ covers $L$, i.e., for every fixed real $\xi \neq 0$ and $(x, t) \in \partial \Omega$ the polynomials $b_{j}(x, t ; \xi, \tau)$ in $\tau(j=1, \ldots, m)$ are linearly independent $\bmod \prod_{j=1}^{m}\left(\tau-\tau_{j}(x, t ; \xi)\right)$.

In $\$ 2$ we shall prove the existence of the Poisson kernels for the system $\left\{L, B_{1}, \ldots, B_{m}, \Omega, \omega\right\}$ (see Theorem 2), using Theorem 1 which is nothing but a simple application of the works of Schechter [7] and [8]. For homogeneous $L, B_{j}$ with constant coefficients and for $\Omega=R_{+}^{n+1}$, the Poisson kernels have been already constructed explicitly by Agmon-Douglis-Nirenberg in [1]. In the last section we consider the Cauchy problem

$$
\begin{aligned}
L u=0 \quad \text { in } t>0, \\
\left(\frac{\partial}{\partial t}\right)^{k} u=\phi_{k}(x) \quad \text { on } \omega, \quad k=1, \ldots, 2 m,
\end{aligned}
$$

and characterize relations between the Cauchy data $\phi_{0}, \ldots, \phi_{2 m-1}$ with which the problem (1) is locally solvable in the direction $t>0$, making use of the Poisson kernels and of the regularity theory stated in the Appendix, where we give a theorem concerning the analytic regularity at the boundary which is a slight improvement of the results of Morrey-Nirenberg [6] (cf. Matsuzawa [4], [5]).

2. Poisson kernels. Throughout this section we suppose that $\Omega, \omega, L$ and $\left\{B_{j}\right\}_{j=1}^{2 m}$ have the same meaning as in the introduction. Let $L^{*}$ be the formal adjoint of $L$ :

$$
L^{*} v=\sum_{|\alpha|+k \leqq 2 m}(-1)^{|\alpha|+k} D_{x}^{\alpha} D_{t}^{k}\left(\bar{a}_{\alpha k} v\right),
$$

where $\bar{a}_{\alpha k}$ is the complex conjugate of $a_{\alpha k}$. Then we can find another Dirichlet set $\left\{B_{j}^{\prime}\right\}_{j=1}^{2 m}$ such that the equality

$$
(L u, v)-\left(u, L^{*} v\right)=\sum_{j=1}^{2 m} \int_{\partial \Omega} B_{j} u\left(B_{2 m-j+1}^{\prime} v\right)^{-} d S, \quad u, v \in C^{\infty}(\bar{\Omega})
$$

holds and $\left\{B_{j}^{\prime}\right\}_{j=1}^{m}$ covers $L^{*}$, where $d S$ is the element of surface and $(u, v)$ is the usual inner product in $L^{2}(\Omega)$ (for the existence of such $\left\{B_{j}^{\prime}\right\}_{j=1}^{2 m}$ see [7]).

For nonnegative integer $s$, we define the norm $\|\cdot\|_{s}$ by

$$
\|u\|_{s}=\left(\sum_{|\alpha|+k \leqq s} \iint_{\Omega}\left|D_{x}^{\alpha} D_{t}^{k} u\right|^{2} d x d t\right)^{1 / 2}
$$

By $H^{s}(\Omega)$ we mean the Hilbert space resulting from the completion of the set $C^{\infty}(\bar{\Omega})$ by the norm $\|\cdot\|_{s}$. By the fact that $\partial \Omega$ is of class $C^{\infty}$ we mean the existence of a finite open covering in $R^{n+1}$ of $\partial \Omega,\left\{U_{k}\right\}$, such that each $\bar{\Omega} \cap U_{k}$ can be mapped in a one-to-one way onto a hemisphere $V_{R}: y_{1}^{2}+\cdots+y_{n}^{2}+\tau^{2}<R^{2}, \tau \geqq 0$, by a mapping $T_{k}$ which together with its inverse is infinitely differentiable and transforms 
$\partial \Omega \cap U_{k}$ onto $\sigma_{R}: y_{1}^{2}+\cdots+y_{n}^{2}<R^{2}$. Let $\left\{\alpha_{k}(x, t)\right\}$ be a set of functions in $C_{0}^{\infty}\left(R^{n+1}\right)$ such that $\operatorname{supp}\left[\alpha_{k}\right] \subset U_{k}$ and $\sum_{k} \alpha_{k}=1$ on $\partial \Omega$. For $g \in C^{\infty}(\partial \Omega)$, we set

$$
\begin{aligned}
g_{k}(y) & =\alpha_{k}\left(T_{k}^{-1}(y, 0)\right) g\left(T_{k}^{-1}(y, 0)\right) & & \text { on } \sigma_{R}, \\
& =0 & & \text { on } R^{n}-\sigma_{R} .
\end{aligned}
$$

For a nonnegative integer $s$, we define

$$
\begin{gathered}
\langle g, h\rangle_{s}=\sum_{k} \int|\xi|^{2 s-1} \hat{g}_{k}(\xi)\left(\hat{h}_{k}(\xi)\right)^{-} d \xi, \quad g, h \in C^{\infty}(\partial \Omega), \\
\langle g\rangle_{s}^{2}=\langle g, g\rangle_{s}, \quad g \in C^{\infty}(\partial \Omega),
\end{gathered}
$$

where $\hat{g}_{k}$ is the Fourier transform of $g_{k}$ :

$$
\hat{g}_{k}(\xi)=\int e^{-i y \xi} g_{k}(y) d y \quad\left(y \xi=y_{1} \xi_{1}+\cdots+y_{n} \xi_{n}\right)
$$

It follows that (3) and (4) satisfy all the properties of an inner product and norm, respectively, and that there exists a constant $C_{s}$ such that, for all $v \in C^{\infty}(\partial \Omega)$ satisfying $v=g$ on $\partial \Omega$,

$$
\langle g\rangle_{s} \leqq C_{s}\|v\|_{s}
$$

Now we can state

THEOREM 1. Let $l$ be an integer satisfying $l>(n+1) / 2$. There then exists a linear mapping $M$ on $H^{l}(\Omega)$ into $H^{l+2 m}(\Omega)$ such that for every $f \in H^{l}(\Omega)$

$$
\begin{aligned}
L(M f) & =f \quad \text { in } \Omega, \\
B_{k}(M f) & =0 \quad \text { on } \omega, \quad k=1, \ldots, m, \\
\|M f\|_{l+2 m} & \leqq C_{l}^{\prime}\|f\|_{l},
\end{aligned}
$$

where $C_{l}^{\prime}$ is a constant not depending on $f$ and it should be noted that $H^{l+2 m}(\Omega)$ $\subset C^{2 m}(\bar{\Omega})$ (cf. Sobolev's lemma).

Proof. We employ the same reasoning as in the proof (of sufficiency) of Theorem 5.2 in [8]. Let $\zeta$ be in $C^{\infty}(\partial \Omega)$ such that $\zeta=0$ on $\omega$ and $\zeta \neq 0$ on $\partial \Omega-\omega$. It then follows from (5) and the coercive inequality for $L^{*},\left\{B_{j}^{\prime}\right\}_{j=1}^{m}$ that the estimate

(8) $c^{-1}\|v\|_{2 m}^{2} \leqq\left\|L^{*} v\right\|_{0}^{2}+\sum_{j=1}^{m}\left\langle B_{j}^{\prime} v\right\rangle_{2 m-\mu_{j}^{\prime}}^{2}+\sum_{j=m+1}^{2 m}\left\langle\zeta B_{j}^{\prime} v\right\rangle_{2 m-\mu_{j}^{\prime}}^{2}+\|v\|_{0}^{2} \leqq c\|v\|_{2 m}^{2}$

holds for all $v \in C^{\infty}(\bar{\Omega})$ and so for all $v \in H^{2 m}(\Omega), c$ being a positive constant not depending on $v$ and $\mu_{j}^{\prime}$ the order of $B_{j}^{\prime}$. The ellipticity of $L^{*}$ and the analyticity of its coefficients guarantee that $v$ vanishes identically in $\Omega$ if it satisfies

$$
\begin{aligned}
& L^{*} v=0 \text { in } \Omega \text {, } \\
& B_{j}^{\prime} v=0 \text { on } \omega \text {, for } j=1, \ldots, m \text {, } \\
& =0 \text { on } \partial \Omega-\omega, \text { for } j=1, \ldots, 2 m \text {, }
\end{aligned}
$$


noting that the set $\left\{B_{j}^{\prime}\right\}_{j=1}^{2 m}$ is a Dirichlet set. Therefore (8) holds without the term $\|v\|_{0}^{2}$.

Application of Lax-Milgram's theorem gives us that for every $f \in H^{l}(\Omega)$ we can find a $w \in H^{2 m}(\Omega)$ such that

$$
\begin{aligned}
{[w, v] } & =\left(L^{*} w, L^{*} v\right)+\sum_{j=1}^{m}\left\langle B_{j}^{\prime} w, B_{j}^{\prime} v\right\rangle_{2 m-\mu_{j}^{\prime}}+\sum_{j=m+1}^{2 m}\left\langle\zeta B_{j}^{\prime} w, \zeta B_{j}^{\prime} v\right\rangle_{2 m-\mu_{j}^{\prime}} \\
& =(f, v), \quad v \in H^{2 m}(\Omega) .
\end{aligned}
$$

Moreover the regularity theory shows us $w \in H^{l+4 m}(\Omega)$ (see [7]). It will then be clear that such a $w$ is uniquely and linearly determined for every $f \in H^{\prime}(\Omega)$. Define $M$ by $M f=L^{*} w$. Then we see easily with the aid of (2) and (9) that $M$ is a linear mapping on $H^{l}(\Omega)$ into $H^{l+2 m}(\Omega)$ and satisfies (6) if $l>(n+1) / 2$.

Next we must prove the continuity of $M$. Taking $v=w$ in (9) we can deduce, from (8) without the term $\| v_{\| 0}^{2}$, the inequality

$$
c^{-1}\|w\|_{2 m}^{2} \leqq(f, w) \leqq c\|w\|_{2 m}^{2} .
$$

Thus we have, with some constant $c^{\prime}>0$,

$$
\|M f\|_{0} \leqq c^{\prime}\|f\|_{0}, \quad f \in H^{l}(\Omega) .
$$

From this we obtain immediately that $M$ is closed, i.e., if a sequence $f_{j}$ converges to $f$ in $H^{l}(\Omega)$ and $M f_{j}$ does to $u$ in $H^{l+2 m}(\Omega)$, then $u=M f$. By the closed graph theorem we can assert that $M$ is a continuous mapping on $H^{l}(\Omega)$ into $H^{l+2 m}(\Omega)$. Thus (7) is obtained. Q.E.D.

Finally we state a theorem on existence of the Poisson kernels:

THEOREM 2. Let $q$ be an integer with the same parity as $n$ satisfying $q>4 m+(n+1) / 2$. There then exist functions $W_{j, q}(x, t ; y)$ in $C^{2 m}\left(\bar{\Omega}_{x, t}\right)(j=1, \ldots, m)$ for every fixed $y$ in $R^{n}$ such that

(i) $D_{x}^{\alpha} D_{t}^{k} W_{j, q}(x, t ; y)$ are continuous in $(x, t ; y) \in \bar{\Omega}_{x, t} \times R_{y}^{n}$ for every $\alpha, k$ satisfying $|\alpha|+k \leqq 2 m$,

(ii) if we regard $W_{j, q}(x, t ; \cdot)$ as a distribution on $R^{n}$ for fixed $(x, t)$, then kernels

$$
K_{j}(x, t ; y)=\Delta_{y}^{(n+q) / 2} W_{j, q}(x, t ; y) \quad(j=1, \ldots, m)
$$

$\left(\Delta_{y}=\sum_{j=1}^{m}\left(\partial / \partial y_{j}\right)^{2}\right)$ are the Poisson kernels for the system $\left\{L, B_{1}, \ldots, B_{m}, \Omega, \omega\right\}$, i.e., for any $\phi_{j} \in C_{0}^{\infty}\left(R^{n}\right)$ the function defined by

$$
u(x, t)=\sum_{j=1}^{m} \int K_{j}(x, t ; y) \phi_{j}(y) d y
$$

satisfies

$$
\begin{aligned}
L u=0 \quad \text { in } \Omega, \\
B_{k} u=\phi_{k} \quad \text { on } \omega, \quad k=1, \ldots, m .
\end{aligned}
$$


Proof. From (7) and Sobolev's lemma we can derive that, if $l>(n+1) / 2$, $H^{2 m+l}(\Omega) \subset C^{2 m}(\bar{\Omega})$ and

$$
|M f|_{2 m, \Omega} \leqq C_{l}^{\prime}\|f\|_{l}, \quad f \in H^{l}(\Omega),
$$

where $C_{l}^{\prime \prime}$ is a constant and

$$
|u|_{s, \Omega}=\sum_{|\alpha|+k \leqq s} \sup _{(x, t) \in \Omega}\left|D_{x}^{\alpha} D_{t}^{k} u(x, t)\right| .
$$

Let $q$ be as in Theorem 2 and $E_{q}(x)$ be a fundamental solution of $\Delta_{x}^{(n+q) / 2}$ belonging to $C^{q-1}\left(R^{n}\right)$ (as such one we can take, following Agmon-Douglis-Nirenberg [1],

$$
-\frac{1}{(2 \pi i)^{n} q !} \int_{|\xi|=1}(x \xi)^{q} \log \frac{x \xi}{i} d \omega_{\xi},
$$

where the principal branch of the logarithm in complex plane slit along the negative real axis is taken, $d \omega_{\xi}$ is the area element on the unit sphere $|\xi|=1$ and $\left.x \xi=x_{1} \xi_{1}+\cdots+x_{n} \xi_{n}\right)$. Since the set $\left\{B_{j}\right\}_{j=1}^{2 m}$ is a Dirichlet set, we can then find $m$ functions $V_{j, q}(x, t ; y)(j=1, \ldots, m)$ such that

(a) $D_{x}^{\alpha} D_{t}^{k} V_{j, q}(x, t ; y)$ are continuous in $(x, t ; y) \in \bar{\Omega}_{x, t} \times R_{y}^{n}$ for all $\alpha, k$ satisfying $|\alpha|+k \leqq q-2 m$,

(b) if we denote by $\delta_{j k}$ the Kronecker delta, then

$$
B_{k} V_{j, q}(x, t ; y)=\delta_{j k} E_{q}(x-y) \quad \text { on } \omega, \quad k=1, \ldots, m .
$$

Put

$$
f_{j, q}^{(y)}(x, t)=-L\left(x, t ; D_{x}, D_{t}\right) V_{j, q}(x, t ; y) .
$$

This and all its derivatives up to order $N=[(n+1) / 2]+1$ are continuous in $\bar{\Omega}_{x, t} \times R_{y}^{n}$. Applying Theorem 1 with $l=N$ to $f(x, t)=f_{j, q}^{(y)}(x, t)$ and putting

$$
H_{j, q}(x, t ; y)=M\left(f_{j, q}^{(y)}\right)
$$

we obtain from (11)

$$
\left|H_{j, q}(\cdot, \cdot ; y)\right|_{2 m, \Omega} \leqq C_{l}^{\prime \prime}\left\|f_{j, q}^{(y)}\right\|_{N} .
$$

From this and (a) we can immediately conclude (i) of Theorem 2 if we put

$$
W_{j, q}(x, t ; y)=V_{j, q}(x, t ; y)+H_{j, q}(x, t ; y) .
$$

Moreover we then have, with (b),

$$
\begin{aligned}
L_{x, t} W_{j, q}(x, t ; y) & =0 & & \text { in } \Omega, \\
B_{k} W_{j, q}(x, 0 ; y) & =\delta_{j k} E_{q}(x-y) & & \text { on } \omega .
\end{aligned}
$$

Let $\phi_{j} \in C_{0}^{\infty}\left(R^{n}\right)(j=1, \ldots, m)$. It then follows from (i) of Theorem 2 and (12) that, for each $j$,

$$
\int K_{j}(x, t ; y) \phi_{j}(y) d y=\int W_{j, q}(x, t ; y) \Delta^{(n+q) / 2} \phi_{j}(y) d y
$$


satisfies

$$
\begin{aligned}
L u & =0 & & \text { in } \Omega \\
B_{k} u & =\delta_{j k} \phi_{j} & & \text { on } \omega, \quad k=1, \ldots, m .
\end{aligned}
$$

This gives the proof of (ii). Q.E.D.

3. Cauchy problem. Let $L, \Omega$ and $\omega$ be the same as those in $\S 1$ and let us take $(\partial / \partial n)^{j-1}(j=1, \ldots, 2 m)$ as boundary operators $B_{j}$ in $\S 1$, where $\partial / \partial n$ is the inner normal derivative to $\partial \Omega$. It is clear that the set $\left\{(\partial / \partial n)^{j-1}\right\}_{j=1}^{2 m}$ is a Dirichlet set. A sequence of integers $\nu=\left(\nu_{1}, \ldots, \nu_{m}\right)$ is said to be admissible if $0 \leqq \nu_{1}<\cdots<\nu_{m} \leqq 2 m-1$ and if the set $(\partial / \partial n)^{v_{1}}, \ldots,(\partial / \partial n)^{v_{m}}$ covers all properly elliptic operators (cf. the notion "completely elliptic" in Hörmander [3]). If $\nu$ is admissible, we can see from $\S 2$ that the Poisson kernels of the form (10):

$$
K_{j}^{(v)}(x, t ; y)=\Delta_{y}^{(n+q) / 2} W_{j, q}^{(v)}(x, t ; y)
$$

for the system $\left\{L,(\partial / \partial n)^{v_{1}}, \ldots,(\partial / \partial n)^{v_{m}}, \Omega, \omega\right\}$ always exist.

TheOREM 3. Let $\nu=\left(\nu_{1}, \ldots, \nu_{m}\right)$ be an admissible sequence. With the Cauchy data $\phi_{0}, \ldots, \phi_{2 m-1}$ in $C_{0}^{\infty}\left(R^{n}\right)$ the Cauchy problem (1) is locally solvable in the direction $t>0$, if and only if $m$ functions

$$
\phi_{v_{k}}(x)-\sum_{j=1}^{m} \int\left(\frac{\partial}{\partial t}\right)^{v_{k}^{\prime}} W_{j, q}^{(v)}(x, 0 ; y) \Delta^{(n+q) / 2} \phi_{v,}(y) d y \quad(k=1, \ldots, m)
$$

are all analytic in $\omega$. Here $\nu_{1}^{\prime}, \ldots, \nu_{m}^{\prime}$ is a sequence such that $0 \leqq \nu_{1}^{\prime}<\cdots<\nu_{m}^{\prime} \leqq 2 m-1$ and $\nu_{k}^{\prime} \neq v_{j}$ for all $k, j$.

Proof. Let $u$ be a solution of (1) with Cauchy data $\phi_{0}, \ldots, \phi_{2 m-1}$ in $C_{0}^{\infty}\left(R^{n}\right)$. Then

satisfies

$$
v=u-\sum_{j=1}^{m} \int K_{j}^{(v)}(x, t ; y) \phi_{v j}(y) d y
$$

$$
\begin{aligned}
L v & =0 \quad \text { in } t>0 \\
\left(\frac{\partial}{\partial t}\right)^{v_{k}} v & =0 \quad \text { on } \omega, \quad k=1, \ldots, m .
\end{aligned}
$$

Thus we can conclude with the aid of the Appendix that $\psi_{k}(x)=(\partial / \partial t)^{v_{k}^{\prime}} v(x, 0)$, that is,

$$
\psi_{k}(x)=\phi_{v_{k}^{\prime}}(x)-\sum_{j=1}^{m} \int\left(\frac{\partial}{\partial t}\right)^{v_{k}^{\prime}} W_{j, q}^{(v)}(x, 0 ; y) \Delta^{(n+q) / 2} \phi_{v_{j}}(y) d y \quad(k=1, \ldots, m)
$$

are all analytic in $\omega$. Conversely, let $\phi_{0}, \ldots, \phi_{2 m-1}$ be arbitrarily given in $C_{0}^{\infty}\left(R^{n}\right)$. If $m$ functions $\psi_{k}(x)(k=1, \ldots, m)$ defined by (13) are all analytic in $\omega$, we can then find a solution of (1). Indeed, let $v$ be a solution of the Cauchy problem

$$
\begin{array}{rlll}
L v=0 & \text { in } t>0, & \\
(\partial / \partial t)^{v_{s} v}=0 & \text { on } \omega, & j=1, \ldots, m, \\
(\partial / \partial t)^{v_{k}^{\prime} v}=\psi_{k} & \text { on } \omega, & k=1, \ldots, m
\end{array}
$$


(we can solve this by the Cauchy-Kowalewski theorem); then we can conclude from (13) that

$$
u=v+\sum_{j=1}^{m} \int W_{j, q}^{(v)}(x, t ; y) \Delta^{(n+q) / 2} \phi_{\nu_{j}}(y) d_{i}
$$

is a solution of (1). The proof is thus complete.

APPENDIX. Let $L\left(x, t ; D_{x}, D_{t}\right)$ be a properly elliptic partial differential operator of order $2 m$ with analytic coefficients defined in a neighborhood of a hemisphere $\Sigma_{\rho}: x_{1}^{2}+\cdots+x_{n}^{2}+t^{2}<\rho^{2}, t>0$, and $B_{1}\left(x ; D_{x}, D_{t}\right), \ldots, B_{m}\left(x ; D_{x}, D_{t}\right)$ be linear partial differential operators of order $\mu_{j}<2 m$ with analytic coefficients defined in the planary boundary of $\Sigma_{\rho}, \sigma_{\rho}: x_{1}^{2}+\cdots+x_{n}^{2}<\rho^{2}$ such that the following are fulfilled:

(i) $\mu_{j} \neq \mu_{k}$ for $j \neq k$,

(ii) if $b_{j}\left(x, D_{x}, D_{t}\right)$ is the part of order $\mu_{j}$ of $B_{j}$, then we have $b_{j}(x ; 0,1) \neq 0$ for all $x \in \sigma_{R}$,

(iii) the set $B_{1}, \ldots, B_{m}$ covers $L$.

Before stating our theorem we have to introduce some notations and to prepare a few lemmas according to Morrey-Nirenberg [6]. For $u \in C^{\infty}\left(\Sigma_{R}\right)(R<\rho)$ and $\phi \in C_{0}^{\infty}\left(R^{n}\right)$ we define two norms, respectively, for $j=0,1, \ldots$,

$$
\begin{aligned}
\left|u ; \Sigma_{R}\right|_{j} & =\left(\iint_{\Sigma_{R}}\left|u^{(j)}\right|^{2} d x d t\right)^{1 / 2}, \\
{[\phi]_{j} } & =\inf \left|v ; R_{+}^{n+1}\right|_{j}\left({ }^{1}\right),
\end{aligned}
$$

where $u^{(j)}$ is a vector function $\left\{D_{x}^{\alpha} D_{t}^{k} u ;|\alpha|+k=j\right\}$ and "inf" is taken over all functions $v$ in $C_{0}^{\infty}\left(\bar{R}_{+}^{n+1}\right)$ which equal $\phi$ on $t=0$. Finally we set

$$
\begin{aligned}
& L^{0}\left(D_{x}, D_{t}\right)=l\left(0,0 ; D_{x}, D_{t}\right), \\
& B_{j}^{0}\left(D_{x}, D_{t}\right)=b_{j}\left(0 ; D_{x}, D_{t}\right),
\end{aligned}
$$

where $l\left(x, t ; D_{x}, D_{t}\right)$ is the leading part of $L$.

The following Lemma is given in [1].

Lemma 1. Assume $u$ is in $C_{0}^{\infty}\left(\bar{R}_{+}^{n+1}\right)$ such that supp $[u] \subset \Sigma_{R} \cup \sigma_{R}(R<\rho)$. Then there exists a constant $K_{1}\left({ }^{2}\right)$ such that

$$
\left|u ; \Sigma_{R}\right|_{2 m} \leqq K_{1}\left(\left|L^{0} u ; \Sigma_{R}\right|_{0}+\sum_{j=1}^{m}\left[B_{j}^{0} u\right]_{2 m-u_{j}}\right) .
$$

(1) We can easily see that this norm is equivalent to one employed in \$2 (cf. (3), (4) and (5)): $\langle\phi\rangle_{j}=\int|\xi|^{2 j-1}|\hat{\phi}(\xi)|^{2} d \xi, \phi \in C_{0}^{\infty}\left(R^{n}\right)$, i.e., there exists a constant $c_{j}$ such that $c_{j}^{-1}\langle\phi\rangle_{j} \leqq[\phi]_{j} \leqq c_{j}\langle\phi\rangle_{,}$, $\phi \in C_{0}^{\infty}\left(R^{n}\right)$.

( ${ }^{2}$ ) From now on we shall use the symbols $K_{i}, K_{i}^{\prime}$ to denote constants depending only on $n, L^{0}$ and $B_{j}^{0}(j=1, \ldots, m)$. 
Lemma 2. Assume $u$ is in $C^{\infty}\left(\Sigma_{R} \cup \sigma_{R}\right)$. There then exists a constant $K_{2}$ such that, for $0<r<r+\delta<R, \delta<r$,

$$
\left|u ; \Sigma_{r}\right|_{2 m} \leqq K_{2}\left(\left|L^{0} u ; \Sigma_{r+\delta}\right|_{0}+\sum_{q=1}^{2 m} \delta^{-q}\left|u ; \Sigma_{r+\delta}\right|_{2 m-q}+\sum_{j=1}^{m}\left[\zeta_{r, \delta} B_{j}^{0} u\right]_{2 m-\mu}\right),
$$

where $\zeta_{r, \delta}(x, t)=h\left(\left(\left(|x|^{2}+t^{2}\right)^{1 / 2}-r\right) / \delta\right)$ with a fixed function $h \in C^{\infty}\left(R^{1}\right)$ satisfying $h(\tau)=1$ for $\tau \leqq 0$ and $h(\tau)=0$ for $\tau \geqq 1$.

Lemma 2 is easily derived from Lemma 1 and the definition of the norm $[\cdot]_{j}$, noting that there exists a constant $C_{k}^{m}$ such that

$$
\sup _{(x, t) \in \Sigma_{R}}\left|\zeta_{r}^{(k)}(x, t)\right| \leqq C_{k}^{m} \delta^{-k}
$$

We need some further notations: for $f, u$ in $\mathrm{C}^{\infty}\left(\Sigma_{R}\right)$

$$
\begin{array}{ll}
M_{R, p}(f)=(p !)^{-1} \sup _{\varepsilon R \leqq r<R}(R-r)^{2 m+p}\left|f ; \Sigma_{r}\right|_{p}, & p=0,1, \ldots, \\
N_{R, p}(u)=[p !]^{-1} \sup _{\varepsilon R \leqq r<R}(R-r)^{2 m+p}\left|u ; \Sigma_{r}\right|_{2 m+p}, & p=-2 m,-2 m+1, \ldots,
\end{array}
$$

and for $v \in C^{\infty}\left(\Sigma_{R} \cup \sigma_{R}\right)$

$$
F_{R, p}^{(j)}(v)=(p !)^{-1} \sup _{\varepsilon R \leqq r<R}(R-r)^{2 m+p}\left[\zeta_{r} v^{(p)}\right]_{2 m-\mu_{j}}, \quad p=0,1, \ldots,
$$

where $\varepsilon=1-1 / 2 m, \zeta_{r}=\zeta_{r, \delta}$ with $\delta=(R-r) /(p+1)$, and $[p !]=p$ ! for $p \geqq 0$ and $[p !]=1$ for $p<0$.

Lemma 3. Suppose $u$ is in $C^{\infty}\left(\Sigma_{R} \cup \sigma_{R}\right)$. Then there exists a constant $K_{3}$ such that, for $p>0$,

$$
N_{R, p}(u) \leqq K_{3}\left(M_{R, p}\left(L^{0} u\right)+\sum_{q=1}^{2 m} N_{R, p-q}(u)+\sum_{j=1}^{m} F_{R, p}^{(j)}\left(B_{j}^{0} u\right)\right) .
$$

This lemma is an immediate consequence of the preceding one.

Now we can prove

THEOREM 4. Let $u$ be a solution in $C^{2 m}\left(\Sigma_{\rho} \cup \sigma_{\rho}\right)$ of the system

$$
\begin{aligned}
L u=f & \text { in } \Sigma_{\rho}, \\
B_{j} u=0 & \text { on } \sigma_{\rho}, \quad j=1, \ldots, m .
\end{aligned}
$$

If $f$ is analytic in a neighborhood of $\Sigma_{\rho}$, then $u$ is analytic on $\Sigma_{\rho} \cup \sigma_{\rho}$.

Proof. To prove this theorem we have only to assert the analyticity of the $u$ in some neighborhood of the origin. It is, first of all, clear that $u$ is in $C^{\infty}\left(\Sigma_{\rho} \cup \sigma_{\rho}\right)$ (see Theorem 15.3 in [1]). As a consequence, if we can establish the following inequalities

$$
N_{R, p}(u) \leqq M \lambda^{p}, \quad R \leqq R_{1} \leqq 1, \quad p=-2 m,-2 m+1, \ldots,
$$

for some fixed constant $M, \lambda \geqq 1$ and $R_{1}<\rho$, we can deduce Theorem 4 from ( $\left.5^{\prime}\right)$ and a slight modification of the proof of Lemma 2.3 in [6]. 
We write the system (4') in the form

$$
\begin{array}{ll}
L^{0} u=f+\left(L^{0}-L\right) u & \text { in } \Sigma_{\rho}, \\
B_{j}^{0} u=\left(B_{j}^{0}-B_{j}\right) u & \text { on } \sigma_{\rho}, \quad j=1, \ldots, m,
\end{array}
$$

and $L^{0}-L, B_{j}^{0}-B_{j}$ in the forms

$$
\begin{aligned}
\left(L^{0}-L\right) u & =\sum_{q=0}^{2 m} a_{q}(x, t) u^{(2 m-q)}, \\
\left(B_{j}^{0}-B_{j}\right) u & =\sum_{q=0}^{\mu_{j}} b_{j, q}(x) u^{\left(\mu_{j}-q\right)},
\end{aligned}
$$

with $a_{0}(0,0)=0$ and $b_{j, 0}(0)=0$. The analyticity of $f, a_{q}$ and $b_{j, q}$ guarantee that we can find numbers $A \geqq 2, H$ and $R_{0} \leqq 1, R_{0}<\rho$, such that

$$
\begin{gathered}
|f|_{p, \Sigma_{R_{0}}} \leqq p ! H A^{p}, \\
\left|a_{q}\right|_{p, \Sigma_{R_{0}}} \leqq p ! H A^{p}, \quad\left|b_{j, q}\right|_{p, \Sigma_{\Sigma_{0}}} \leqq p ! H A^{p}, \\
\left|a_{0}(x, t)\right| \leqq H A(|x|+|t|), \quad\left|b_{j, 0}(x)\right| \leqq H A|x| \text { in } \Sigma_{R_{0}} .
\end{gathered}
$$

Application of Lemma 3 gives us, with the aid of $\left(6^{\prime}\right)$ and $\left(7^{\prime}\right)$, that if $R \leqq R_{0}$, for all $p>0$

$$
\begin{aligned}
N_{R, p}(u) \leqq K_{3}\left(M_{R, p}(f)+\sum_{q=0}^{2 m} M_{R, p}\left(a_{q} u^{(2 m-q)}\right)\right. & +\sum_{q=1}^{2 m} N_{R, p-q}(u) \\
& \left.+\sum_{j=1}^{m} \sum_{q=0}^{\mu_{j}} F_{R, p}^{(j)}\left(b_{j, q} u^{\left(\mu_{j}-q\right)}\right)\right)
\end{aligned}
$$

First we find, with the aid of $\left(2^{\prime}\right),\left(8^{\prime}\right)$ and Leibniz' formula that for $R \leqq R_{0}$ and $p>0$

$$
\begin{aligned}
M_{R, p}(f) \leqq & H K_{1}^{\prime} R^{2 m+(n+1) / 2}(A R)^{p} \\
\sum_{q=0}^{2 m} M_{R, p}\left(a_{q} u^{(2 m-q)}\right) \leqq & H A R N_{R, p}(u)+H \sum_{t=1}^{p}\left(\frac{A R}{2 m}\right)^{t} N_{R, p-t}(u) \\
& +H \sum_{q=1}^{2 m} \sum_{t=0}^{p} A^{-q}\left(\frac{A R}{2 m}\right)^{q+t} N_{R, p-q-t}(u) .
\end{aligned}
$$

From $\left(3^{\prime}\right)$ we have, recalling the definition of the norm $[\cdot]_{j}$,

$$
F_{R, p}^{(j)}\left(b_{j, q} u^{\left(\mu_{j}-q\right)}\right) \leqq(p !)^{-1} K_{2}^{\prime} \sup _{\varepsilon R \leqq r<R}(R-r)^{2 m+p}\left|\zeta_{r}\left(b_{j, q} u^{\left(\mu_{j}-q\right)}\right)^{(p)} ; \Sigma_{r+\delta}\right|_{2 m-\mu_{j}} \cdot
$$

Using $\left(1^{\prime}\right),\left(8^{\prime}\right)$ and Leibniz' formula we obtain, for $j, q$ and $r$ such that $1 \leqq j \leqq m$, $0 \leqq q \leqq \mu_{j}$ and $\varepsilon R \leqq r<R$,

$$
\begin{aligned}
I_{r}^{(j, q)} & =\left|\zeta_{r}\left(b_{j, q} u^{\left(\mu_{j}-q\right)}\right)^{(p)} ; \Sigma_{r+\delta}\right|_{2 m-\mu_{j}} \\
& \leqq K_{3}^{\prime} \sum_{k=0}^{2 m-\mu_{j}} \delta^{-k} \sum_{t=0}^{p+2 m-\mu_{j}-k}\left(\begin{array}{c}
p+2 m-\mu_{j}-k \\
t
\end{array}\right)\left|b_{j, q}^{(t)} u^{(p+2 m-k-t-q)} ; \Sigma_{r+\delta}\right|_{0}
\end{aligned}
$$


From this we obtain, for $p>0$,

$$
\begin{aligned}
(p !)^{-1} & \sup _{\delta R \leqq r<R}(R-r)^{2 m+p} I_{r}^{(j, 0)} \\
& \leqq e\left(1+\frac{1}{p}\right)^{2 m} H\left\{\sum_{k=0}^{2 m-\mu_{j}}(k+1)^{k}\left(A R N_{R, p-k}(u)+\sum_{t=1}^{2 m+p-\mu_{j-k}}(A R)^{t} N_{R, p-k-t}(u)\right)\right\}
\end{aligned}
$$

and when $q \geqq 1$

$$
\begin{aligned}
(p !)^{-1} & \sup _{\varepsilon R \leqq r<R}(R-r)^{2 m+p} I_{r}^{(j, q)} \\
& \leqq e\left(1+\frac{1}{p}\right)^{2 m} H \sum_{k=0}^{2 m-\mu j}(k+1)^{k} \sum_{t=0}^{2 m+p-\mu_{j}-k} A^{-q}(A R)^{t+q} N_{R, p-k-t-q}(u) .
\end{aligned}
$$

Consequently

$$
\begin{aligned}
\sum_{j=1}^{m} \sum_{q=0}^{\mu_{j}} F_{R, p}^{(j)}\left(b_{j, q} u^{\left(\mu_{j}-q\right)}\right) & \\
& \leqq K_{4}^{\prime}\left(H A R \sum_{k=0}^{2 m} N_{R, p-k}(u)+H \sum_{k=0}^{2 m} \sum_{\tau=1}^{2 m+p-k}(A R)^{z} N_{R, p-k-\tau}(u)\right) .
\end{aligned}
$$

It follows from $\left(9^{\prime}\right),\left(10^{\prime}\right)$ and $\left(11^{\prime}\right)$ that

$$
\begin{aligned}
& N_{R, p}(u) \leqq K_{4}\left(H R^{2 m+(n+1) / 2}(A R)^{p}\right.+H A R N_{R, p}(u)+H A R \sum_{k=1}^{2 m} N_{R, p-k}(u) \\
&\left.+H \sum_{k=0}^{2 m} \sum_{\tau=1}^{2 m+p-k}(A R)^{\tau} N_{R, p-k-\tau}(u)+\sum_{k=1}^{2 m} N_{R, p-k}(u)\right) .
\end{aligned}
$$

Hence, if we set

$$
R_{1}=\min \left(\frac{1}{2 K_{4} H A}, R_{0}\right),
$$

the inequality

$$
\begin{aligned}
N_{R, p}(u) \leqq & 2 K_{4}\left(H R^{2 m+(n+1) / 2}(A R)^{p}+H \sum_{k=0}^{2 m} \sum_{\imath=1}^{2 m+p-k}(A R)^{\imath} N_{R, p-\imath-k}(u)\right) \\
& +\left(1+2 K_{4}\right) \sum_{k=1}^{2 m} N_{R, p-k}(u)
\end{aligned}
$$

holds for $R \leqq R_{1}$ and $p>0$.

Using $\left(12^{\prime}\right)$ we can finally establish the inequality $\left(5^{\prime}\right)$ with

$$
\begin{aligned}
\lambda & =6+6 K_{4}+3 A R_{1}, \\
M & =2 K_{4} H R_{1}^{2 m+(n+1) / 2}+\lambda^{2 m}\left|u ; \Sigma_{R_{1}}\right|_{2 m} .
\end{aligned}
$$

Thus the proof is completed. 


\section{BIBLIOGRAPHY}

1. S. Agmon, A. Douglis and L. Nirenberg, Estimates near the boundary for solutions of elliptic partial differential equations satisfying general boundary conditions. I, Comm. Pure Appl. Math. 12 (1959), 623-727.

2. J. Hadamard, Le problème de Cauchy, Hermann, Paris, 1932.

3. L. Hörmander, On the regularity of the solutions of boundary problems, Acta Math. 99 (1958), 225-264.

4. T. Matsuzawa, Regularity at the boundary for solutions of hypo-elliptic equations, Osaka J. Math. 3 (1966), 313-334.

5. - On quasi-elliptic boundary problems, Trans. Amer. Math. Soc. 133 (1968), 241-265.

6. C. B. Morrey, Jr. and L. Nirenberg, On the analyticity of the solutions of linear elliptic systems of partial differential equations, Comm. Pure Appl. Math. 10 (1957), 271-290.

7. M. Schechter, General boundary value problems for elliptic partial differential equations, Comm. Pure Appl. Math. 12 (1959), 457-486.

8. - Various types of boundary conditions for elliptic equations, Comm. Pure Appl. Math. 13 (1960), 407-425.

Aichi University of EdUCATION,

OKAZAKI-SHI, AICHI-KEN, (JAPAN) 